BMC

Biophysics



Hierarchical super-structure identified by polarized light microscopy, electron microscopy and nanoindentation: Implications for the limits of biological control over the growth mode of abalone sea shells

Schneider et al. 


\title{
Hierarchical super-structure identified by polarized light microscopy, electron microscopy and nanoindentation: Implications for the limits of biological control over the growth mode of abalone sea shells
}

Andreas S Schneider ${ }^{1+}$, Birgit Heiland ${ }^{1}$, Nicolas J Peter ${ }^{1}$, Christina Guth ${ }^{1}$, Eduard Arzt ${ }^{1,2}$ and Ingrid M Weiss ${ }^{1,3^{*+}}$

\begin{abstract}
Background: Mollusc shells are commonly investigated using high-resolution imaging techniques based on cryo-fixation. Less detailed information is available regarding the light-optical properties. Sea shells of Haliotis pulcherina were embedded for polishing in defined orientations in order to investigate the interface between prismatic calcite and nacreous aragonite by standard materialographic methods. A polished thin section of the interface was prepared with a defined thickness of $60 \mu \mathrm{m}$ for quantitative birefringence analysis using polarized light and LC-PolScope microscopy. Scanning electron microscopy images were obtained for comparison. In order to study structural-mechanical relationships, nanoindentation experiments were performed.

Results: Incident light microscopy revealed a super-structure in semi-transparent regions of the polished cross-section under a defined angle. This super-structure is not visible in transmitted birefringence analysis due to the blurred polarization of small nacre platelets and numerous organic interfaces. The relative orientation and homogeneity of calcite prisms was directly identified, some of them with their optical axes exactly normal to the imaging plane. Co-oriented "prism colonies" were identified by polarized light analyses. The nacreous super-structure was also visualized by secondary electron imaging under defined angles. The domains of the super-structure were interpreted to consist of crystallographically aligned platelet stacks. Nanoindentation experiments showed that mechanical properties changed with the same periodicity as the domain size.

Conclusions: In this study, we have demonstrated that insights into the growth mechanisms of nacre can be obtained by conventional light-optical methods. For example, we observed super-structures formed by co-oriented nacre platelets as previously identified using X-ray Photo-electron Emission Microscopy (X-PEEM) [Gilbert et al., Journal of the American Chemical Society 2008, 130:17519-17527]. Polarized optical microscopy revealed unprecedented super-structures in the calcitic shell part. This bears, in principle, the potential for in vivo studies, which might be useful for investigating the growth modes of nacre and other shell types.
\end{abstract}

\footnotetext{
* Correspondence: ingrid.weiss@inm-gmbh.de

${ }^{\dagger}$ Equal contributors

'INM - Leibniz Institute for New Materials, Campus D2 2, 66123,

Saarbruecken, Germany

${ }^{3}$ Universität Regensburg, Biochemie I, Regensburg 93053, Germany

Full list of author information is available at the end of the article
} 


\section{Background}

In the need for optimizing the materials performance, hierarchical construction principles became genetically manifest in the growth processes of skeleton forming animals [1] over millions of years of evolution [2-6]. The mollusk shell is one of the most ancient examples of such a hierarchical material [7-11]. Shells often consist of different crystalline polymorphs of calcium carbonate minerals such as calcite and aragonite, which are embedded in organic components [12-14]. Commonly known structures are nacre, and prismatic and crossed-lamellar shell types $[15,16]$. Many aspects regarding the optical and mechanical properties of mollusk shells are well understood $[12,17]$. The most studied shell type is nacre [18-21].

Since it is difficult to precisely classify the shell structures according to crystal sizes [22], it is more appropriate to characterize natural composites according to volume fractions of organic and inorganic phases. In the case of sea shells, the mineral part makes up more than $95 \mathrm{wt} \%$ of the natural composite $[2,23]$. The relationships between structural and mechanical properties of nacre were identified on different length scales [11,22,24]. This shell type consists of mineral tablets of about $0.5-1 \mu \mathrm{m}$ in thickness and about $5-10 \mu \mathrm{m}$ in diameter. Its fracture toughness is relatively high $(\sim 7 \mathrm{MPa} \sqrt{\mathrm{m}})$ as compared to the inorganic compound $(\sim 0.2 \mathrm{MPa} \sqrt{\mathrm{m}})$, whereas the hardness is in a similar range, $\sim 200-300 \mathrm{H}_{\mathrm{v}}$, for both nacre and aragonite [25]. In this respect, nacre differs from other biological minerals such as corals $\left(\sim 0.1 \mathrm{MPa} \sqrt{\mathrm{m}}, 5-10 \mathrm{H}_{\mathrm{v}}\right)$ and calcitic egg shells [25].

However, the growth mechanisms of the different shell structures remain speculative due to the lack of highresolution light optical techniques, which are required to study shell formation in vivo, and the semi-transparency of adult shells. A seminal SEM study was performed by Wise [26]. He reported the sheet-like structure of the organic matrix at the nacreous growth front of the gastropod Cittarium pica and discussed the formation of crystal stacks in the light of earlier suggestions for their columnar growth mode in Haliotis [26] (and refs. therein). Wise pointed out that this is in clear contrast to the horizontal layer-by-layer growth modes for pelecypod (bivalve) nacre, e.g. of Pinctada radiata. The morphological study of Wise [26] could not answer the question regarding whether or not the organic matrix acts as a template for crystal nucleation by epitaxy. Weiner and Traub were using X-ray diffraction and demonstrated the perpendicular orientation of some organic molecules such as chitin fibers [27] with respect to the c-axes of the aragonite crystals for gastropods, bivalves and cephalopods. In the case of Nautilus the degree of misorientation of the organic matrix was in the same order of magnitude as for the a and b plane of the aragonite crystals [28].
When these authors used electron diffraction in order to gradually reduce the sampling areas down to approximately the size range of a single biological cell, they identified locally ordered aragonite patterns but, on larger scales and depending on the biological species, a mosaic organization of the organic matrix with various degrees of order [29]. This suggests a gradual, kinetic selection of faster growth rates and a small probability of nucleating a new orientation. Recent X-PEEM synchrotron experiments demonstrated a high degree of order within stacks of abalone nacre vs. a considerable degree of disorder towards the neighbouring stacks [1]. The stacks of crystalline aragonite tablets in nacre are misoriented in their $\mathrm{c}$-axis with respect to each other and orientational ordering of tablet stacking occurs not abruptly but gradually over a distance of $50 \mu \mathrm{m}[1,30]$. Initially, the orientation of nucleation sites could be completely random, as shown e.g. in Figure 8 of [21]. It can be assumed that the orientation of the first platelet existing next to the prismatic layer is determined by the roughness of the substrate (e.g. calcite prism, spherulitic aragonite, ACC precursor granules). It is well-known that nucleation sites are located within a gel-like organic matrix $[10,31]$ and, that certain aragonite-inducing and chitin-binding proteins may form an oriented 3-dimensional nucleation site $[27,32]$. Although the distribution and function of such nucleation sites have not yet been identified on the molecular level and many aspects are still under debate [33], a detailed texture analysis of calcite shells using EBSD suggests that similar mechanisms are valid for molluscs in general and also for members of other phyla such as brachiopods [34,35].

The present study was motivated by the challenge to improve quantitative light-optical investigations of mollusk shells, which would be of particular interest because they bear the potential for in vivo experiments. We performed quantitative polarization analysis using liquid crystal compensator technology (LC-PolScope Abrio ${ }^{\mathrm{TM}}$ Imaging System), introduced in 1995 by Oldenbourg $[36,37]$. The quality and reproducibility of LC-PolScope data depends not only on sample thickness but also on structural features [38], which are in the range of the wavelength of light and below $[39,40]$. Hence, the strategy used here involved sample preparation according to standard metallographical methods for producing thin sections of defined thickness and orientation [41]. In parallel to LC-PolScope analysis, a detailed lightmicroscopic investigation of composite interfaces using materials polarization microscopy was performed. We also characterized the mechanical properties of a polished section of the nacre/prismatic (N/P) shell transition region using nanoindentation. Due to the high lateral resolution of nano-indentation, we were able to correlate the observed structural features to mechanical function. 


\section{Results}

\section{Incident light microscopy}

The transition region between the outer prismatic calcite part and the inner aragonite (nacre) part of a Haliotistype sea shell (Additional file 1: Figure S1) was prepared using standard metallographic procedures (Figure 1).

Shells were embedded in two different orientations: For orientation A, the polished section was horizontal to the coronal plane of the shell (Figure 1, left), which results in an inclination angle between the normal of each nacre platelet and the normal of the polished surface. This angle is related to the natural curvature of the Haliotis specimen and also depends on how much of the shell material was removed by the grinding and polishing procedure. For orientation $\mathrm{B}$, the polished surface is a transverse section (Figure 1, right). In this case, the normal of each nacre platelet is perpendicular to the normal of the polished surface.

The polished cross-sections were characterized using an incident light microscope. As shown in Figure 2, the sample orientation has a strong influence on the optical

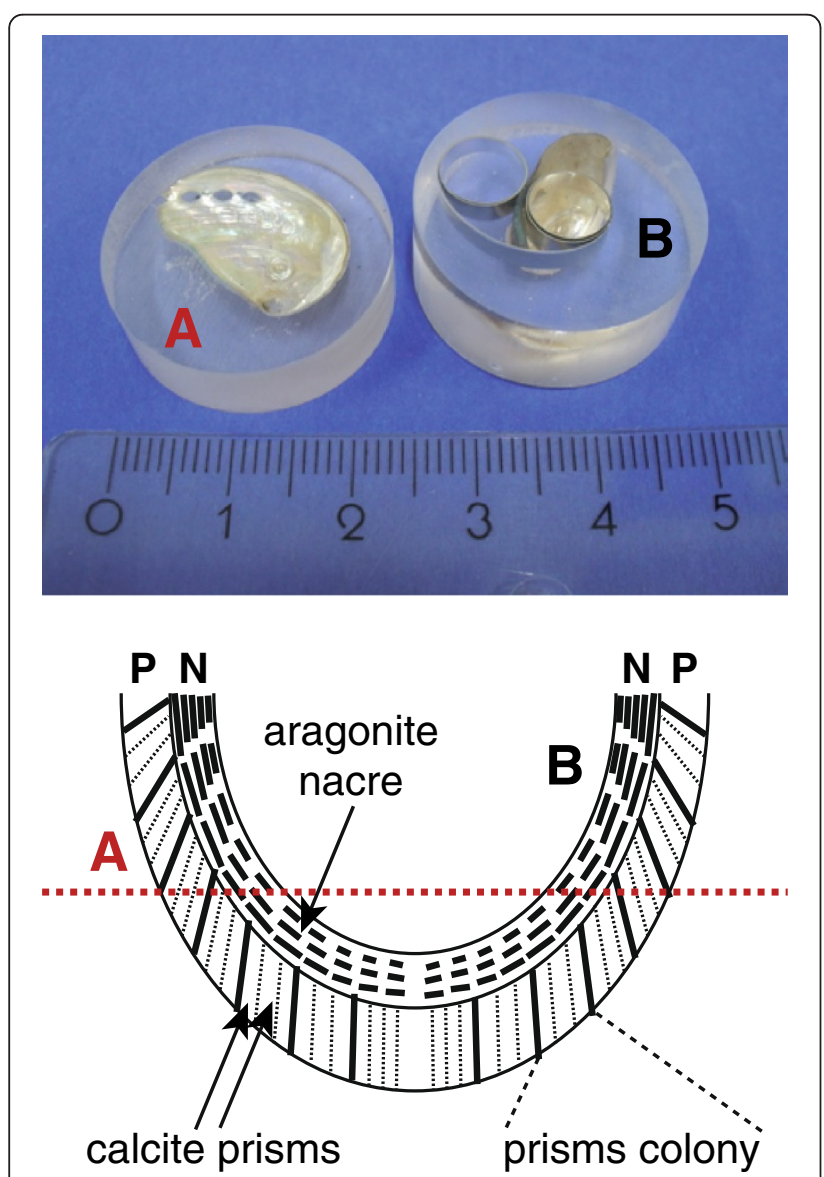

Figure 1 Sample preparation. Metallographic preparation of Haliotis pulcherina sea shell. Left, orientation $\mathbf{A}$; right, orientation $\mathbf{B}$. appearance of the two shell parts (nacre and prismatic) in the bright-field imaging mode. The lamellar structure of the nacreous part in the $\mu \mathrm{m}$ range is only visible in orientation A (Figure $2 \mathrm{a}, \mathrm{c}$ ), whereas the prismatic calcite part is characterized by structures in the sub-mm range which are best visualized in orientation B (Figure $2 b, d$ ). Due to the inclination angle of the nacre platelets with respect to the surface, single lamellae exceed the resolution limit of the optical microscope only in orientation A.

Besides the well-known regular nacre layers, we observed undefined colored reflexes with mosaic arrangement and dimensions covering several platelets in a stacked configuration in the incident light optical image, which cannot be corelated with surface topography. In the following, we refer to this additional contrast, which covers multiple platelets, as super-structure. (Figure 2c, Additional file 2: Figure S2).

It is known that each nacre layer reflects and transmits part of the incident white light, which causes constructive and destructive interference of the light. Due to the extinction of certain parts of the visible spectrum nacre occurs in different colors if viewed from different directions. Therefore, the iridescent properties of nacre are basically explained by Bragg's law:

$$
2 d \sin \theta=n \lambda
$$

where $d$ is the distance between reflecting planes, $\theta$ is the diffraction angle, $n$ is the refractive index and $\lambda$ is the wavelength of radiation. In case of nacre, where the aragonite layers are separated by organic interfaces in the size range of $10-100 \mathrm{~nm}, d$ is approximately the thickness of one aragonite layer. The diffraction angle $\theta$ is defined as the angle between the incident light and the reflecting nacre planes and thus depends on the orientation of the nacre platelets with respect to the polished surface.

We do not expect that the orientation as well as the thickness of the nacre platelets vary on a length scale of the super-structure. Therefore, according to Equation (1) the local color contrast can only be assigned to local changes in the refractive index, which is related to the orientation-sensitive dielectric constant of the material. The color contrast does not appear in orientation B (Figure 2b), since the reflective planes (aragonite layers) are oriented parallel to the beam and thus the Bragg condition is not fulfilled.

At this stage of the experiments it remained unclear whether the mineral crystals or the organic interfaces contribute significantly to this effect.

In order to investigate the nature of the superstructure, we chose a comparative approach using different light and electron microscopic techniques for the specimen with orientation A. 

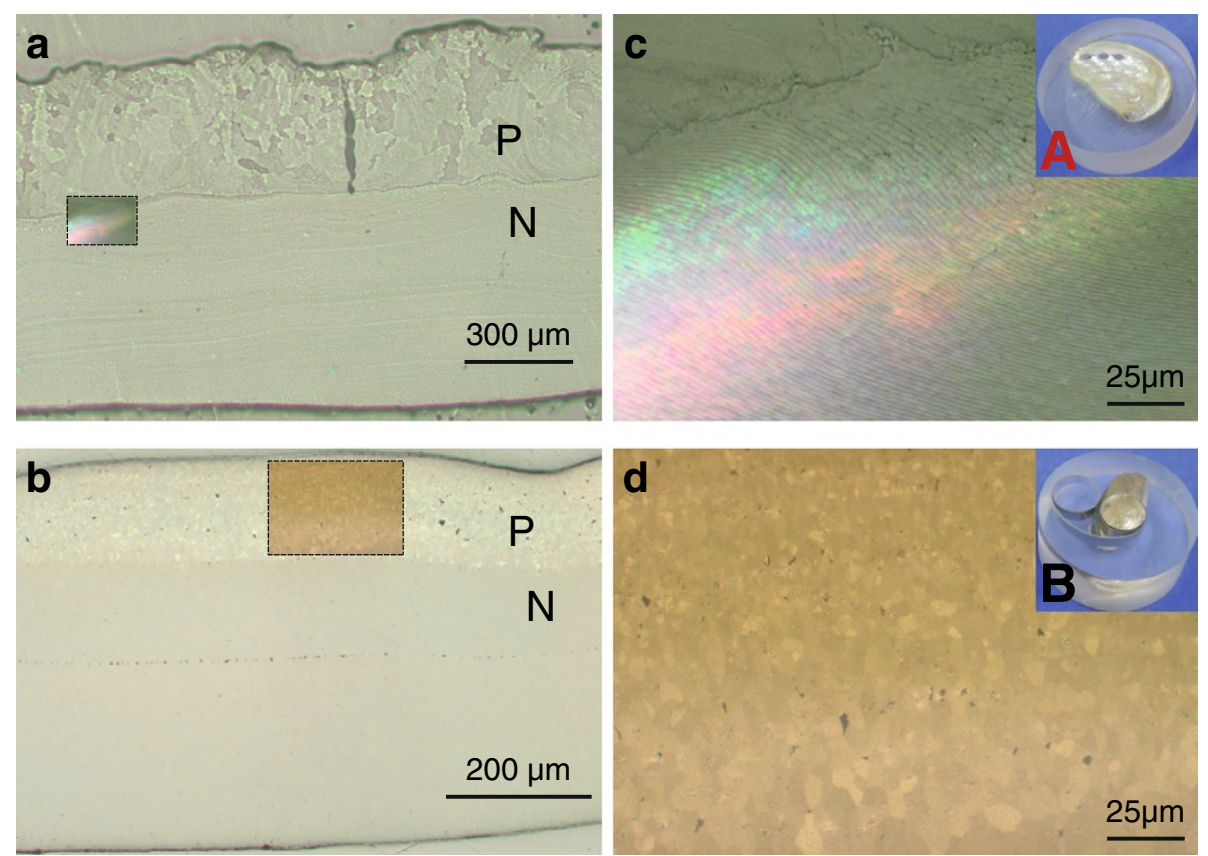

Figure 2 Incident light microscopy of differently oriented shell cross-sections. Incident light microscopy. Top, orientation $\mathbf{A}$; bottom, orientation B. Hierarchical structures of nacreous and prismatic shell parts are visualized best in orientation A and B, respectively. Inserts in the images on the left side are shown at higher magnification on the right. Note that in addition to the nacre lamellae there is another level of hierarchy in the range of $\sim 20 \mu \mathrm{m}$, defined here as the super-structure, which is observed in orientation $\mathrm{A}$ at higher magnification. P, prismatic layer; N, nacre.

\section{Transmitted light microscopy}

For these experiments, a $60 \mu \mathrm{m}$ thin section of the abalone shell in orientation A was prepared. In contrast to incident light microscopy, a clear structure of the calcite layer is visible in transmitted polarized light microsopy. It consists of domains of different sizes in the range of 5-100 $\mu \mathrm{m}$, which appear in different colors (Figure 3). The contrast in polarized light microscopy is directly related to the 3-dimensional orientation of the unit cell of anisotropic crystals [12]. Closer inspection of the large domains reveals that these also consist of subunits in the range of $5 \mu \mathrm{m}$, which we interpret as the individual prisms. A rotation of the specimen by $90^{\circ}$ did not cause an equal color change for all prisms (Figure $3 a$ vs b). The colors of some prisms change with respect to their direct neighbours, which indicates that they have different crystallographic orientations. If the color of adjacent prisms changes in the same way, one can conclude that their relative orientations are the same. Since the prisms are inclined with respect to the surface, it remains difficult to predict the relative crystallographic orientation. Some of the prisms at the interface between calcite and aragonite remain dark (Figure 3), although no defects are present in the thin section. This suggests that these prisms have an amorphous structure or, that calcite crystals are oriented exactly with their optical axis perpendicular to the surface. The stacking of adjacent prisms with equal crystallographic orientation seems to be a structural feature of the calcitic shell part and is in the following referred to as prism colony.

Further, the previously observed lamellar structure of the nacre (Figure 2c, Additional file 2: Figure S2) is not visible in Figure 3 (N, nacreous shell part). One would expect a strong crystalline birefringence signal for aragonite for this area. However, we could not observe any structure in the sense that well-defined boundaries could be identified. This suggests that the number of interfaces in the sampled volume is too large [12]. In our case, we roughly estimate that the investigated specimen consists of $\sim 60$ nacre platelets interspaced by organic sheets across the transmitted section. Another explanation would be that the signals originating from crystallographically misaligned aragonite platelets are superimposed, hence causing the blurred polarization.

\section{LC-PolScope birefringence analysis}

LC-PolScope Abrio ${ }^{\text {TM }}$ analysis was performed to further investigate the birefringence of calcite and aragonite structures in this $60 \mu \mathrm{m}$ thin section of the Haliotis shell in transmission. Figure 4 shows the calculated birefringent retardance (Figure 4a) and orientation (Figure 4b) images of the same area as shown in Figure 3. The prism colonies in the calcite layer have almost the same 

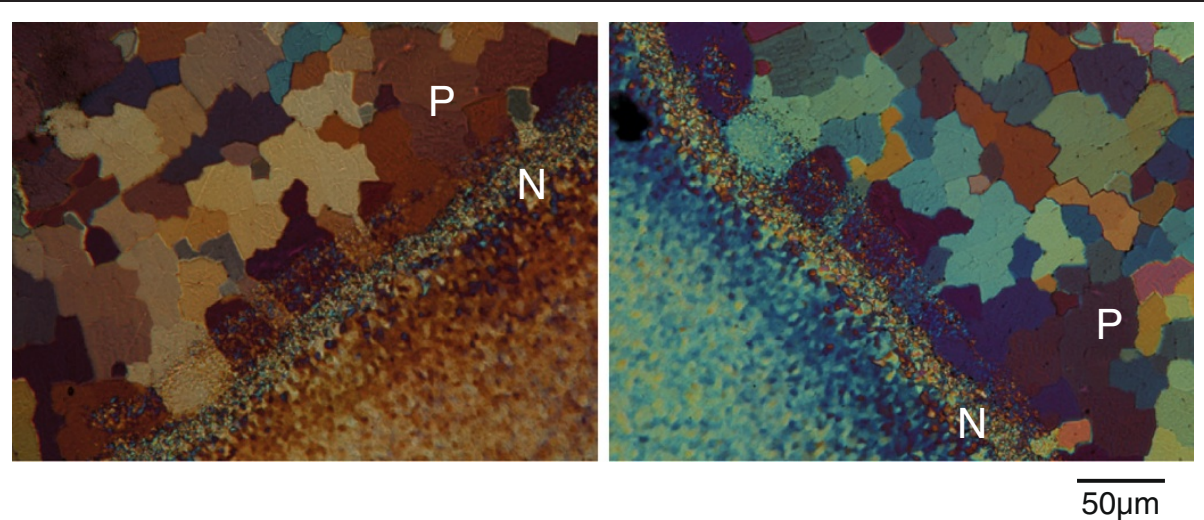

Figure 3 Transmitted light microscopy of Haliotis shell thin section. Polarized light microscopy in transmission of a $60 \mu \mathrm{m}$ thin section in orientation A. The transition region nacre/prismatic is centred on the microscopy stage. The left and the right image were obtained at the same position by rotating the stage $90^{\circ}$. The structure of the prismatic layer $(\mathrm{P})$ is clearly visible. The tapering nacre structure gradually changes as a function of distance from the prismatic layer. Note that neither the lamellar structure nor the super-structure are visible. P, prismatic layer; N, nacre.

morphologies as observed by conventional transmitted polarized light microscopy (Figure 3).

The orientation image (Figure $4 \mathrm{~b}$ ) indicates that the prisms cover a large range of crystallographic orientations and that adjacent prisms in a single colony have more or less similar crystallographic orientations. For differently oriented colonies the birefringent retardance values of prisms and colonies were in different ranges (e.g. $109 \mathrm{~nm}$ in blue areas, $267 \mathrm{~nm}$ in red areas; Figure 4a, arrows). For example, the prism colony with an azimutal angle of $17^{\circ}$ (color coded in orange and indicated also by the array overlay of azimutal angles in Figure $4 \mathrm{~b}$ ) has a retardance value of $109 \mathrm{~nm}$, whereas the prism colony with an azimutal angle of $129^{\circ}$ (color coded in purple) revealed a retardance of $267 \mathrm{~nm}$. In contrast to synthetic calcite [42], further comparison of Figures $4 \mathrm{a}$ and b shows that areas of homogeneous orientation are not necessarily related to homogeneous birefringent retardance values of the prisms. This could be due to the fact that prisms are not pure single crystals of calcite but consist of complex arrangements of organic and inorganic phases $[43,44]$. The interface between adjacent colonies and also adjacent prisms within the colonies appeared with nonuniform colors in both, retardance and orientation mode.
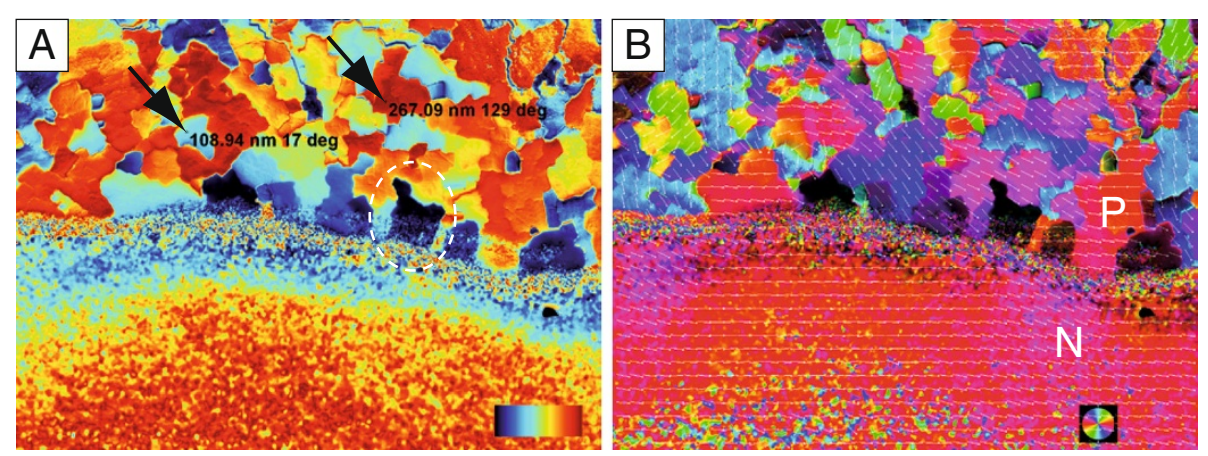

$\overline{100 \mu m}$

Figure 4 LC-PolScope analysis of nacre/prismatic transition. LC-PolScope ("Abrio ${ }^{\mathrm{TM}}$-Imaging") microscopy in transmission of a $60 \mu \mathrm{m}$ section in orientation A. The birefringent retardance values (left, 0-273 nm from dark blue to dark red) and the orientation of the slow axis vector (right, red $0^{\circ} / 180^{\circ}$, light blue $90^{\circ} / 270^{\circ}$ ) are calculated for each pixel and color coded in the images. The two arrows in the left image indicate two differently oriented calcite prisms. Note that the marked light blue prism with a retardance value of $\sim 109 \mathrm{~nm}$ appears orange in the orientation image (right), which corresponds to an azimutal angle of $17^{\circ}$. The marked red prism with a retardance value of $\sim 267 \mathrm{~nm}$ appears purple in the orientation image (right), which corresponds to an azimutal angle of $129^{\circ}$. This analysis shows that the prisms consist of different domains, which can be distinguished according to their homogeneous crystallographic orientation. Not all of them are homogeneous. The tapering nacre layer gradually changes as a function of distance from the prismatic layer. Due to the blurred polarization signal, no structure is visible. Arrows in the left image indicate pixels with medium (ligh blue, $109 \mathrm{~nm} / 17^{\circ}$ ) and high (dark red, $267 \mathrm{~nm} / 129^{\circ}$ ) birefringent retardance contrast. P, prismatic layer; N, nacre. 
This can be attributed to the organic layers surrounding the prisms [43]. Finally, the same colonies which did not change their color after $90^{\circ}$ rotation in the conventional polarized light microscopy analysis exhibit low birefringent retardance (dark areas, Figure 4a, dotted circle) and could therefore not be identified with respect to orientation. These areas were identified by Raman imaging spectroscopy to consist of calcite (Additional file 3: Figure S3).

Although the thin section is too thick in order to quantify the birefringence in the bulk of the nacre layer, the tapering overlap region with the calcite layer can be used to obtain information regarding the orientation of individual platelets. On top of the prisms with zero retardance (Figure $4 \mathrm{a}$, dotted circle), individual nacre platelets or fragments of the tapered nacreous layer were identified. Raman imaging spectroscopy confirmed that these platelets consist of aragonite (Additional file 3: Figure S3). These nacre parts appear in the blue range of retardance values. At areas where the nacreous layer is thicker and composed of several platelets stacked on the prisms, the retardance reaches the yellow-red range. This shows that the thickness of the nacreous layer significantly contributes to the observed retardance contrast. In areas where the thin section consists only of the nacreous part, a gradient of retardance values from light blue to red was detected. This phenomenon cannot directly be correlated to the structure of the sea shell and may be caused by the multiple superpositions of differently oriented nacre platelets with internal structures (Additional file 4: Figure S4 shows isolated nacre platelets investigated by LC-PolScope microscopy). Furthermore, the large fraction of interfaces contributes to form birefringence which complicates data analysis.

\section{Scanning electron microscopy}

The structure of the nacre/prisms transition region in orientation A was additionally investigated by scanning electron microscopy. As shown in Figure 5a, the nacre layer is composed of domains which differ in their grey levels. These domains encompass several stacked nacre platelets, which appear with identical grey levels. These platelet stacks are in the size range of the super-structure as observed by incident light microscopy (Figure 2c, Additional file 2: Figure S2).

At higher magnification (Figure 5b,c) it can be seen that the domain boundaries are related to the boundaries of individual nacre platelets. From the literature it is known that the c-axis orientation of the aragonite platelets is not necessarily perpendicular with respect to the plane of each individual layer, but varies $[1,33,45]$. Accordingly, the different grey levels of the domains might be explained by an electron channeling contrast caused by the differently oriented areas. It is well known that
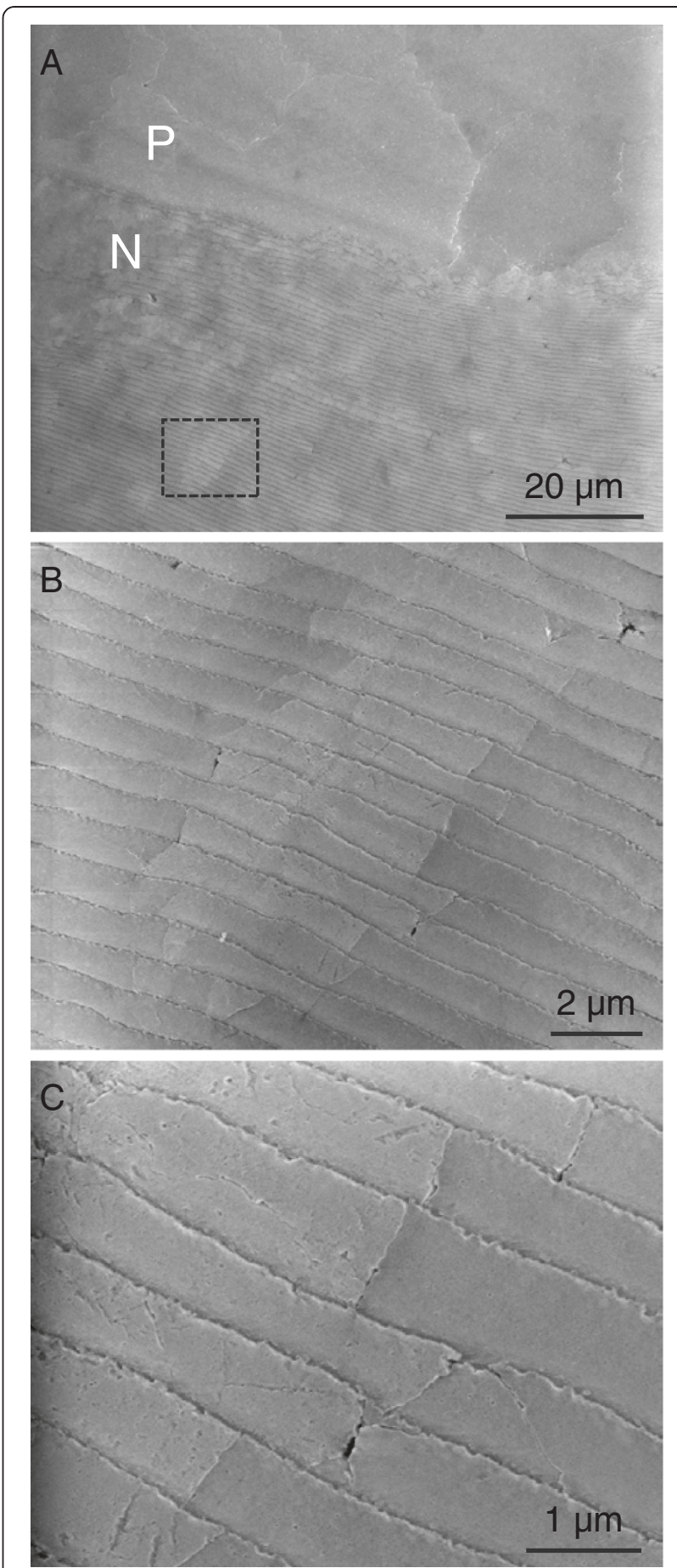

Figure 5 Scanning electron microscopy image of the nacre super-structure. Scanning electron microscopy of a $60 \mu \mathrm{m}$ section in orientation A. Top, overview image showing the transition between prismatic and nacreous layers. Center, super-structure in the size range of $20 \mu \mathrm{m}$ in the nacreous layer. Domains of homogeneous grey level are formed by stacks of adjacent nacre platelets. Bottom, boundary between two domains which form the super-structure. 
backscattered electrons (BE) provide information about crystallographic orientation via channeling [46]. Secondary electron (SE) images also contain this channeling contrast, though mostly superimposed by a stronger topography contrast. The LFD detector used for imaging in low vacuum collects a broader spectrum of electrons as compared to the conventional Everhard-Thorneley detector. Therefore, both $\mathrm{SE}$ and $\mathrm{BE}$ contribute to the contrasting of the image, which might explain the orientation sensitiviy of the SEM experiments with uncoated specimens. Tilting enhances the contrast as it increases the yield of backscattered electrons, reduces multiple scattering and energy loss processes and enhances channeling [46].

The fact that the super-structure was observed by two different contrasting methods, i.e. incident light microscopy and scanning electron microscopy, indicates that surface topography effects can be ruled out. The superstructure must rather be attributed to the biological growth mode [1].

\section{Nanoindentation}

In order to investigate the implication of the superstructure on the mechanical properties of the shell, nanoindentation experiments were performed on a polished section in orientation B. This orientation was chosen as in this case the nacre platelets are parallel to the indentation axis, which makes data analysis more straight forward. The indents were performed with a maximum load of $10 \mathrm{mN}$ and a distance between adjacent indents of $20 \mu \mathrm{m}$. Furthermore, the indents were positioned in two parallel rows such that they cover a certain part of the calcite and the nacre layer.

Figures $6 \mathrm{a}$ and $\mathrm{b}$ show the mechanical properties of the sea shell as a function of indent position. For both rows the first indents were placed in the calcite layer and all other indents were positioned in the nacreous part. Hardness and Young's modulus vary significantly over the investigated area, especially across the interface. For the calcite prisms, hardness values of about 4.2 to $5.2 \mathrm{GPa}$ and Young's moduli of approximately $72 \pm$ $2 \mathrm{GPa}$ were found. For the nacre layer, lower values of 2.5 to $3.7 \mathrm{GPa}$ and about $65 \pm 2 \mathrm{GPa}$ were found. In addition, it was observed that both hardness and Young's modulus slightly increase in the nacre layer from the interface calcite - aragonite to the inner surface of the shell.

Another observation in Figure 6 is the systematic variation in hardness and Young's modulus for calcite and aragonite. As the distance between adjacent indents is similar to the characteristic size of the previously observed super-structure, this variation could reflect the different properties of individual domains of the superstructure. This is demonstrated in Figure 6c, which shows an SEM image of residual indents in the polished
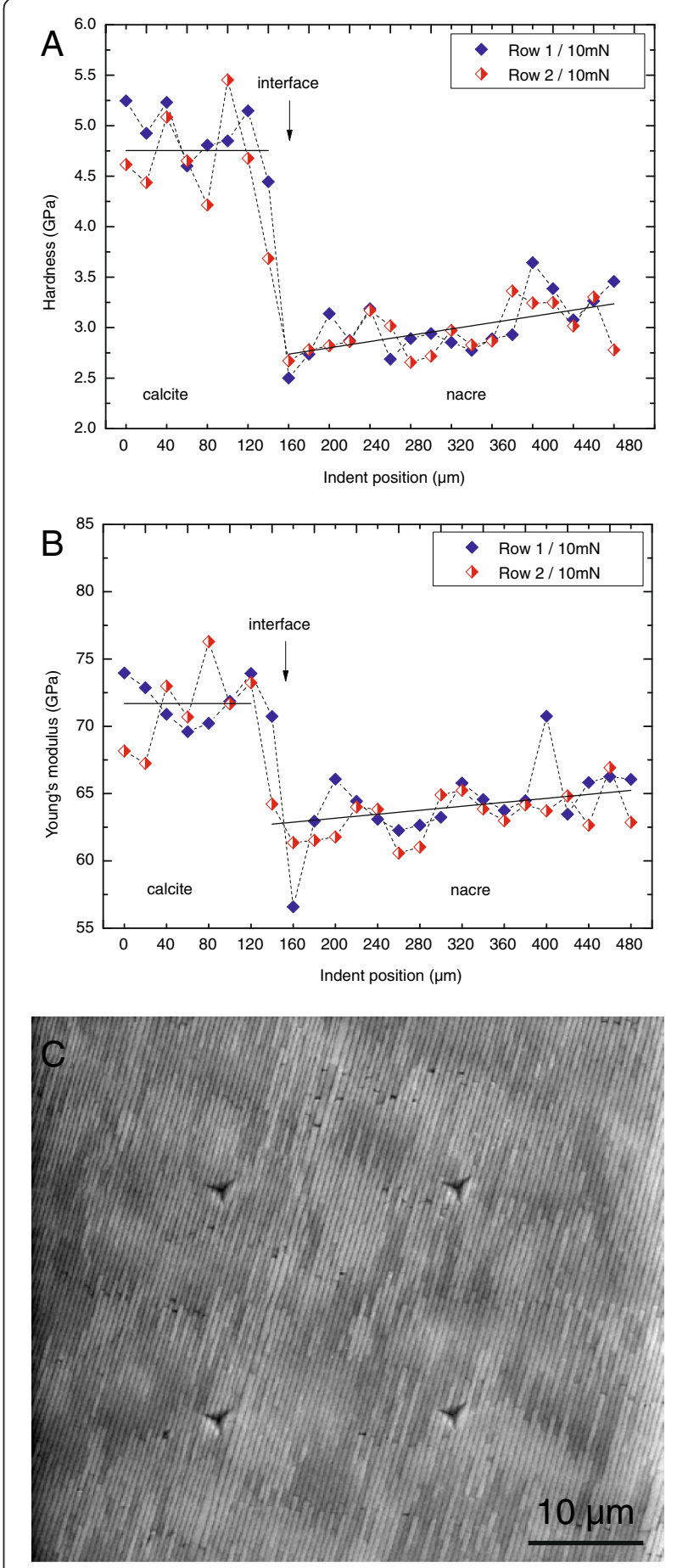

Figure 6 Nanoindentation of Haliotis shell sectioned in orientation B. Nanoindentation experiments performed on a polished section in orientation B. Hardness (A) and Young's modulus (B) are shown as a function of indentation position. C, Scanning electron microscopy image of the cross section after the experiments. The positions of some indents with respect to the $20 \mu \mathrm{m}$ super-structure are exposed. Indents were $20 \mu \mathrm{m}$ apart from each other and do not exactly match with the periodicity of the super-structure. Note that the super-structure in this image was visualized at a tilt angle of $20^{\circ}$. 
section. The image was obtained under a defined tilt angle of $20^{\circ}$ in order to visualize the super-structure in the SEM even for the sample in orientation B. Note that indents were randomly distributed over areas (platelet stacks) with different grey levels.

\section{Discussion}

Here we report the investigation of carefully prepared polished sections and a semi-transparent thin-section of the nacre-prismatic transition region of Haliotis pulcherina shells. The combination of conventional incident light optical microscopy, quantitative polarized light microscopy (LC-PolScope Abrio $^{\mathrm{TM}}$ system) in transmission, VP-SEM and nanoindentation enabled the detection of superstructures in both, nacre and prismatic shell regions. In Haliotis rufescens nacre, a similar structure was previously identified by X-PEEM [1]. Actually, we applied the same light microscopy and VP-SEM methods to the nacreous layer of the bivalve Mytilus and observed also a superstructure comparable to the one in Haliotis pulcherina (Figure 7). Observing the same feature in both, gastropods and bivalves, with completely different growth modes in nacre [31], raises the question whether this structural feature is evolutionary advantageous, or whether it is the result of mineralization processes. For example, the experimental observations made by Gilbert et al. [1] agreed well with kinetic growth models [30]. On the other hand, for other controlled biomineralization systems such as bone hydroxyapatite and sponge silica, the formation of distinct super-structures are mechanically advantageous [47]. Accordingly, super-structures can be an evolutionary advantage and therefore genetically conserved "on



Figure 7 Scanning electron microscopy image of nacre super-structures. Scanning electron microscope image of a Mytilus galloprovincialis sea shell. Domains of homogeneous grey level are formed by stacks of adjacent nacre platelets. purpose" in mollusc species in general, regardless of their particular growth modes.

The biological control mechanisms are well understood on the level of a single nacre platelet [31]. However, mechanisms explaining the overall structure of shells are still not clear. With respect to the super-structure observed here, we suggest the following scenario: The formation of the very first nacre layer starts at the irregular growth front with the deposition of nucleation seeds [21], which are most likely specific proteins secreted by the tissue. Hence, the nacre platelets originating from these seeds will be randomly oriented with respect to their aragonite c-axis. Since the growth kinetics is directly related to the crystallographic orientation of the growing crystal, it is more likely that platelets with a prefered orientation grow on the expense of less well oriented platelets and thus occupy more space in a given time. Once another layer is deposited and new seed crystals start to grow, those growing on top of the "well oriented" platelets, which occupy already a much larger space than the less well oriented ones, have a higher probability to grow faster and with the prefered orientation. Although mineral bridges could contribute to transfer crystal orientation on the level of adjacent nacre platelets within one stack of platelets, they can not account for the super-structure, which is composed of differently oriented platelet stacks. On the level of competing stacks of platelets, the orientation, concentration and arrangement of nucleation seeds would be predetermined by the organic matrix.

The same may apply for prisms, which finally appear as prism colonies with aligned orientations of adjacent crystals.

In order to see whether the super-structures affect the mechanical properties, which would be an evolutionary advantage $[47,48]$, indentation experiments were performed on the size scale of the super-structure.

Overall, hardness and Young's modulus values found for Haliotis pulcherina nacre are very similar to those recently reported [49-52]. Using microindentation, for example Fleischli et al. [52] obtained a hardness of 3.9 GPa for Haliotis rufescencs. The Young's modulus in their study was only measured by nanoindentation. At low penetration depths, an average value of $72 \mathrm{GPa}$ was detected. However, it was realized that with increasing penetration depth, the Young's modulus droped significantly. For example, at a penetration depth of $160 \mathrm{~nm}$ they found a modulus of $65 \mathrm{GPa}$, which is very similar to the results of the current study. The strong influence of the penetration depth in [52] was explained by the indentation size effect and an increasing contribution of the soft organic matrix with increasing penetration depth. Note that for geological aragonite without any polymer inclusions the nanohardness is in a range of $6.2 \pm 0.3 \mathrm{GPa}$ 
and Young's modulus is in the order of $102.8 \pm 2.4 \mathrm{GPa}$ [53].

The influence of the organic matrix may also contribute to the different mechanical properties of the calcite and aragonite layer. Both layers consist of mineralized calcium carbonate and a small fraction of biopolymer. However, in the regular brick and mortar structure of the nacre, each calcium carbonate platelet is surrounded by a soft matrix on a length scale, which is sensed by nanoindentation. Therefore, in nacre the organic phase has to carry the same load as the hard particles, which causes the lower hardness and Young's values compared to calcite. The influence of the organic layer can be estimated by simple composite models [54] or, more realistically, by the "shear lag model" [55].

The gradual change of the mechanical properties in the nacre layer is likely related to changes of structural features along the cross-section of the sample. In particular, Pokroy et al. [56] have shown that the thickness of the nacre platelets increases from the interface calcite/ aragonite to the inner surface of the shell. Similar changes in structural features were observed by Nudelman et al. [18] at the growth front of nacre. Therefore, it can be assumed that the contribution of the softer organic matrix to the mechanical properties decreases as the positions of the indents approach the inner surface. This explains the higher hardness and Young's modulus values for indents performed closer to the inner surface of the shell.

The systematic variation in hardness and Young's modulus for calcite and aragonite, might partially be explained by topography effects, or locally different contributions of the soft organic matrix. However, also the crystallographic orientation of the indented structural features might contribute to this effect. Our experiments confirm the original observations made by Schmidt [12], who showed that individual prisms in the calcite layer have different crystallographic orientations. Therefore, the scatter in the Young's modulus can be explained by the fact, that indents were performed in prisms with a soft or hard crystallographic direction $[57,58]$.

For the nacre layer, where the size of the structural features is smaller than the indented area, the influence of the crystallographic orientation is less obvious. Normally it would be expected that the influence of the crystallographic orientation of the platelets is averaged out, if several platelets are indented at the same time. However, the super-structure observed in this work and the work by Gilbert et al. [1] indicate that groups of adjacent platelets have an equal crystallographic orientation. Thus the scatter in the indentation data likely reflects the different orientations of these tablet stacks and is an example of where the observed super-structure influences the mechanical properties of sea shell.
All methods applied in this study enable a fast and easy screening of different types of mollusc shells. This is important to identify different growth modes as a function of cell physiology and biochemistry. Here we successfully demonstrated using combined methods that both, nacre and prismatic shell parts consists of characteristic superstructures at the nacre-prismatic interface. We also demonstrate that the super-structures correlate with distinct mechanical properties. These results allow us to draw conclusions regarding the limits of biological control.

\section{Conclusions}

Extensive studies targeted the crystallographic orientation of aragonite platelets in the nacreous layer and calcite prisms in the prismatic layer with respect to the overall shape and function of mollusc shells [12,17]. However, hierarchical super-structures have to the best of the authors knowledge not yet reported to be obtained with conventional light optical and SEM techniques, only with much more sophisticated methods such as synchrotron X-PEEM and TEM. Here, we identified an additional contrast in the incident light microscopy and interpret this as additional nacre and prismatic superstructures. Both super-structures encompass several subunits of the structure such as nacre platelets and calcite prisms. Our observations have major implications for the growth mode, the limits of biological control, and the potential consequences for shell mechanics in various species and developmental stages. This bears an enormous potential for in vivo studies using conventional optical techniques, which might be useful to shed new light on the growth modes of nacre, the prismatic interface and other shell types in real time.

\section{Methods}

\section{Sample preparation}

Haliotis pulcherina shells of $10-20 \mathrm{~mm}$ diameter were obtained commercially (U.S. Shell Inc., Los Fresnos, Texas, U.S.A.). We followed established procedures for sample processing [59-61]. In brief, shell pieces were mounted in two different orientations at room temperature in epoxy resin (Epofix, Struers, Willich, Germany) using a vacuum impregnation device (Cast N'Vac-1000, Buehler, Düsseldorf, Germany).

Flat cross-sections were obtained by $\mathrm{SiC}$ paper grinding at grain sizes of 46, 26, 15, and $8 \mu \mathrm{m}$ (Buehler, Düsseldorf, Germany), subsequent polishing with "Dur" cloths and abrasives of $6 \mu \mathrm{m}$ and $3 \mu \mathrm{m}$, and "Mol" cloths and abrasives of $1 \mu \mathrm{m}$ (Struers, Willich, Germany), respectively. Specimens were endpolished using "NAP" cloths and OP$\mathrm{S}$ suspension (Struers), rinsed extensively with water and ethanol, and air dried. The quality of the cross-sections and the cleanliness of the surfaces were confirmed in reflected bright field mode. 
Thin sections were obtained by mounting the polished flat cross-section onto a glass slide using the epoxy resin (Epofix, Struers, Willich, Germany) and abrasing the epoxy resin from the top towards the shell to a final specimen thickness of $60 \mu \mathrm{m}$. The final thin section was again endpolished.

\section{Incident light microscopy}

Reflected bright field mode microscopy was performed using an inverted research metallurgical microscope PMG3 (Olympus, Hamburg, Germany) equipped with NeoSPlan 10 NIC $0.25 \infty /-\mathrm{f}=180$, NeoSPlan 20 NIC $0.4 \infty / 0$ $\mathrm{f}=180$ and NeoSPlan 50 NIC $0.7 \infty / 0 \mathrm{f}=180$ objectives. Images were recorded at $2080 \times 1544$ pixel resolution with the Olympus Digital Color Camera UC30 and "analySIS start" (Olympus Soft Imaging Solutions GmbH; www.olympus-sis.com; Münster, Germany).

This microscope with the same objectives, and with crossed polarizers and Nicol's prism was used for quality control of polished surfaces in DIC (differential interference contrast) mode.

\section{Transmitted light microscopy}

Transmitted light microscopy was performed using a wide-field polarization microscope Orthoplan-Pol (Leitz, Wetzlar, Germany) equipped with NPL FLUOTAR $16 \times / 0.45 \mathrm{P}$ and NPL FLUOTAR $25 \times / 0.55 \mathrm{P}$ objectives. Images were recorded with an external 7.5 MPixel Live View Digital SLR Camera (E-330 ZUIKO DIGITAL 14-45 mm 1:3.5-5.6 ; Olympus Imaging Corp., www. olympus-europa.com) with a C-mount adaptor. The camera settings for taking the electronic images were optimized according to the best match with manual adjustments for visual analysis.

\section{LC-PolScope birefringence analysis}

An LC-PolScope image processing system (CRI, LOTOriel, Darmstadt, Germany) was used for quantitative birefringence analysis at $546 \mathrm{~nm}$ using the standard Abrio $^{\mathrm{TM}}$ filter. The LC-PolScope camera was mounted on a Zeiss inverted microscope (Zeiss Observer Z1, Göttingen, Germany) equipped with A-Plan $10 \times / 0.25 \mathrm{Ph} 1, \mathrm{LD}$ Plan Neofluar 20x/0.4 Korr Ph2, LD Plan Neofluar $40 \times / 0.6$ Korr Ph2, and Plan Achromat $63 \times / 1.40$ Oil DIC objectives. All images were obtained according to the manufacturers instructions. Background images were taken from a selected area of the transparent glas slide without the biological sample. The Abrio ${ }^{\mathrm{TM}}$ image processing software (CRI/LOT-Oriel; [62,63]) was used for quantitative data evaluation per pixel with respect to both, the retardance mode and the orientation of the slow optical axis (azimuth) mode.

\section{Raman imaging microscopy}

The mineralogy of the specimens was investigated at room temperature in air using a confocal LabRAM ARAMIS Vis Raman Imaging Spectrometer (Horiba Jobin Yvon, Bensheim, Germany), equipped with $50 \times / 0.75$ $\mathrm{WD}=0.37 \mathrm{~mm}$ air objective Olympus MPlan (Olympus, Germany), and a diode laser $785 \mathrm{~nm}, 80 \mathrm{~mW}$ (Pilot P500, Sacher Lasertechnik Group, Marburg, Germany). Spectra represent the average of 2 measurements with an exposure time of $4 \mathrm{sec}$.

\section{Scanning electron microscopy (VP-SEM)}

Specimens were investigated without any coating in lowvacuum mode at $100 \mathrm{~Pa}$ using a FEI Quanta VP-SEM (FEI, Eindhoven, Netherlands), equipped with a secondary electon detector, ETD Everhard-Thornley-Detector and a LFD Large-Field-Detector for low vacuum imaging. Images were obtained at $10 \mathrm{keV}$ and $15 \mathrm{keV}$ (working distance 7 and $10 \mathrm{~mm}$ ) at room temperature.

\section{Mechanical testing, nanoindentation}

Nanoindentation experiments were performed on polished sections of shell specimens using a Hysitron Triboindenter TI 950 equipped with a standard transducer and a Berkovich tip. In total, two rows of 25 indents with peak loads of $10 \mathrm{mN}$ were applied along the cross-section of the shell. The distance between two adjacent indents was $20 \mu \mathrm{m}$ and tests were run in open-loop mode. For the extraction of hardness and Young's modulus from the data, the unloading segment of the curves was analyzed by the Oliver and Pharr method [64].

\section{Isolation of single nacre platelets}

Abalone shells (Haliotis pulcherina) were rinsed with tap water and dried with clean tissue. The outer prismatic calcite shell layers were mechanically and chemically removed using $0.6 \mathrm{M} \mathrm{HCl}$. The remaining acid was completely removed with pure water and dried in air. Nacreous shell parts were treated with sodium hypochlorite solution (12\% active chlorine) for 24 hours and the platelet fraction was recovered from the turbid supernatant by 2 min. centrifugation at $3000 \mathrm{rpm}$ (Rotanta $460 \mathrm{RS}$ with swing out rotor, Hettich, Germany) at room temperature. Subsequent washing steps of the solid phase were performed 20x with pure water until any chlorine vapour was completely removed.

\section{Additional files}

Additional file 1: Fractured shell at the nacre/prismatic transition Left image, Environmental scanning electron microscopy image of a fractured Haliotis pulcherina sea shell. The sharp transition from prismatic calcite (top layer) to aragonite nacre (bottom layer) is formed by differentiated shell forming regions of the mantle epithelial tissue. The 
biological regulation of such sharp transitions between different shell structures is still enigmatic. Right image, scanning electron microscopy image of the nacreous part of a fractured Haliotis pulcherina sea shell. The regular stacking of single nacre platelets is visualized, whereas a superstructure in the size range of up to 20 single platelets in vertical direction is not observed here.

\section{Additional file 2: Incident light microscopy of nacre shell part.}

Incident light microscopy of polished shell cross-section in orientation A (compare also Figure 2C). In addition to the nacre lamellae there is another level of hierarchy in the range of $\sim 20 \mu \mathrm{m}$, defined here as the super-structure, which is observed in orientation A at higher magnification.

\section{Additional file 3: Raman imaging microscopy of Haliotis shell thin} section. The transition region contains overlapping calcite and aragonite phases, which can be clearly identified by Raman imaging spectroscopy. Raman spectra of two different calcite regions are shown. Prisms with high birefringent retardance values were identified as calcite (red spectrum). The prisms which appeared black with zero retardance in LC-PolScope microscopy are clearly identified as calcite (purple / dark blue spectrum). Aragonite spectra were obtained in tapering nacre shell parts close to the transition region, partly with some calcite finger-print (blue-green, light blue spectra). Spectra were not background subtracted with respect to the epoxy resin (green).

Additional file 4: Birefringent retardance of isolated nacre platelets. LC-PolScope orientation image of isolated nacre tablets from Haliotis pulcherina sea shell. In addition to single nacre platelets (arrow), clusters of platelets attached to one another in a fragment of a single nacre layer are also visible. The overall birefringence in the $\mathrm{c}$-axis direction of each platelet is weak, whereas the form birefringence at the boundary between mineral and water gives a bright contrast. This is demonstrated by the fact that confluent platelets show internal boundaries between single platelets with only weak retardance values. Dot-like structures are also visible, which have similar orientations within the imaging plane (a- and b-axis of the aragonite crystals) in certain domains of each platelet. The dot-like patterns could be internal or surface structures. If they are internal structures, they could be organic matrix shielded by the mineral phase or, replica voids as a result of sodium hypochlorite treatment, which was used to solubilize the interlamellar organic matrix. They could as well be the result of surface topography.

\section{Competing interests}

The authors declare that they have no competing interests.

\section{Authors' contributions}

BH carried out the metallographic specimen preparation and light optical analysis. ASS and NJP carried out the electron microscopy experiments and mechanical testing. CG participated in the specimen preparation and performed spectroscopy measurements. BH and EA participated in the interpretation of data. ASS and IMW conceived of the study, and participated in its design and coordination, and interpretation of data. ASS, BH, EA and IMW drafted the manuscript. All authors read and approved the final manuscript.

\section{Acknowledgements}

The authors thank Roland Bennewitz and Marcus Koch for constructive comments on the manuscript, and Caroline Bylda and Sarah Schmidt for technical assistance.

\section{Author details}

'INM - Leibniz Institute for New Materials, Campus D2 2, 66123,

Saarbruecken, Germany. ${ }^{2}$ Saarland University, Saarbruecken 66123, Germany.

${ }^{3}$ Universität Regensburg, Biochemie I, Regensburg 93053, Germany.

Received: 2 April 2012 Accepted: 3 September 2012

Published: 12 September 2012

\section{References}

1. Gilbert PUPA, Metzler RA, Zhou D, Scholl A, Doran A, Young A, Kunz M, Tamura N, Coppersmith SN: Gradual ordering in red abalone nacre. J Am Chem Soc 2008, 130:17519-17527.

2. Currey JD, Taylor JD: The mechanical behaviour of some molluscan hard tissues. J Zool 1974, 173:395-406.

3. Lowenstam HA, Weiner S: On Biomineralization. New York: Oxford Univ Press; 1989.

4. Simkiss K, Wilbur KM: Biomineralization: Cell Biology and Mineral Deposition. San Diego, CA (U.S.A.): Academic; 1989.

5. Dunlop JWC, Fratzl P: Biological composites. Annu Rev Mater Res 2010, 40:1-24.

6. Wegst UGK, Ashby MF: The mechanical efficiency of natural materials. Philos Mag 2004, 84:2167-2181.

7. Mann S: Biomineralization. Principles and concepts in bioinorganic materials chemistry. New York: Oxford Univ. Press; 2001.

8. Meldrum FC, Cölfen H: Controlling mineral morphologies and structures in biological and synthetic systems. Chem Rev 2008, 108:4332-4432.

9. Weiner S, Mahamid J, Politi Y, Ma Y, Addadi L: Overview of the amorphous precursor phase strategy in biomineralization. Front Mater Sci Chin 2009, 3:104-108.

10. Addadi L, Joester D, Nudelman F, Weiner S: Mollusk shell formation: a source of new concepts for understanding biomineralization processes. Chem - A Eur J 2006, 12:980-987.

11. Barthelat F: Nacre from mollusk shells: a model for high-performance structural materials. Bioinspir Biomim 2010, 5:035001.

12. Schmidt WJ: Die Bausteine des Tierkörpers in Polarisiertem Lichte. Bonn: Cohen; 1924.

13. Towe KM, Hamilton $\mathrm{GH}$ : Ultrastructure and inferred calcification of the mature and developing nacre in bivalve mollusks. Calcif Tissue Int 1967, 1:306-318.

14. Rousseau M, Lopez E, Stempflé P, Brendlé M, Franke L, Guette A, Naslain R, Bourrat X: Multiscale structure of sheet nacre. Biomaterials 2005, 26:6254-6262.

15. Bøggild OB: The shell structure of the mollusks. K Dan Vidensk Selsk Skr Naturvidensk Math Afd 1930, 9:233-326.

16. Taylor JD, Kennedy WJ, Hall A: The shell structure and mineralogy of the bivalvia. Bull Br Mus Nat Hist (Zool) 1973, 22:253-294.

17. Currey JD: Mechanical Properties of Mollusc Shell. In The mechanical properties of biological materials. Cambridge, U.K: Cambridge Univ. Press; 1980.

18. Nudelman F, Shimoni E, Klein E, Rousseau M, Bourrat X, Lopez E, Addadi L, Weiner S: Forming nacreous layer of the shells of the bivalves Atrina rigida and Pinctada margaritifera: An environmental- and cryo-scanning electron microscopy study. J Struct Biol 2008, 162:290-300.

19. Jäger $C$, Cölfen H: Fine structure of nacre revealed by solid state ${ }^{13} \mathrm{C}$ and ${ }^{1} \mathrm{H}$ NMR. CrystEngComm 2007, 9:1237-1244.

20. Mukai H, Saruwatari K, Nagasawa H, Kogure T: Aragonite twinning in gastropod nacre. J Cryst Growth 2010, 312:3014-3019.

21. Zaremba CM, Belcher AM, Fritz M, Li Y, Mann S, Hansma PK, Morse DE, Speck JS, Stucky GD: Critical transitions in the biofabrication of abalone shells and flat pearls. Chem Mater 1996, 8:679-690.

22. Barthelat F, Li CM, Comi C, Espinosa HD: Mechanical properties of nacre constituents and their impact on mechanical performance. J Mater Res 2006, 21:1977-1986

23. Sarikaya M, Gunnison KE, Yasrebi M, Aksay IA: Mechanical propertymicrostructural relationships in abalone shell. MRS Proc 1989, 174:109.

24. Meyers MA, Lin AYM, Chen PY, Muyco J: Mechanical strength of abalone nacre: Role of the soft organic layer. J Mech Behav Biomed Mater 2008, 1:76-85

25. Ashby MF: The CES EduPack database of natural and man-made materials. In Granta Material Inspiration - Bioengineering. Edited by Design G. Cambridge, U.K: Granta Design; 2008.

26. Wise SW: Microarchitecture and deposition of gastropod nacre. Science 1970, 167:1486-1488.

27. Levi-Kalisman Y, Falini G, Addadi L, Weiner S: Structure of the nacreous organic matrix of a bivalve mollusk shell examined in the hydrated state using Cryo-TEM. J Struct Biol 2001, 135:8-17.

28. Weiner $S$, Traub W: X-ray diffraction study of the insoluble organic matrix of mollusk shells. FEBS Lett 1980, 111:311-316. 
29. Weiner S, Talmon Y, Traub W: Electron diffraction of mollusc shell organic matrices and their relationship to the mineral phase. Int I Biol Macromol 1983, 5:325-328

30. Coppersmith SN, Gilbert PUPA, Metzler RA: Theoretical characterization of a model of aragonite crystal orientation in red abalone nacre. J Phys $A$ Math Theor 2009, 42:125101.

31. Nudelman F, Gotliv BA, Addadi L, Weiner S: Mollusk shell formation: Mapping the distribution of organic matrix components underlying a single aragonitic tablet in nacre. J Struct Bio/ 2006, 153:176-187.

32. Suzuki M, Saruwatari $K$, Kogure T, Yamamoto $Y$, Nishimura T, Kato T, Nagasawa $\mathrm{H}$ : An acidic matrix protein, Pif, is a key macromolecule for nacre formation. Science 2009, 325:1388-1390.

33. Checa AG, Cartwright JHE, Willinger M-G: Mineral bridges in nacre. J Struct Biol 2011, 176:330-339.

34. Hahn S, Rodolfo-Metalpa R, Griesshaber E, Schmahl WW, Buhl D, Hall-Spencer JM, Baggini C, Fehr KT, Immenhauser A: Marine bivalve geochemistry and shell ultrastructure from modern low $\mathrm{pH}$ environments. BGD Biogeosci Discuss 2011, 8:10351

35. Goetz AJ, Steinmetz DR, Griesshaber E, Zaefferer S, Raabe D, Kelm K, Irsen S, Sehrbrock A, Schmahl WW: Interdigitating biocalcite dendrites form a 3-D jigsaw structure in brachiopod shells. Acta Biomater 2011, 7:2237-2243.

36. Oldenbourg R: Polarization microscopy with the LC-PolScope. In Live Cell Imaging: A Laboratory Manual. Edited by Goldman RD, Spector DL. New York: Cold Spring Harbor Laboratory Press; 2004:205-237.

37. Oldenbourg R, Mei G: New polarized light microscope with precision universal compensator. J Microsc 1995, 180:140-147.

38. Eder M, Lütz-Meindl U, Weiss IM: Non-invasive LC-PolScope imaging of biominerals and cell wall anisotropy changes. Protoplasma 2010, 246:49-64.

39. Alberts B, Johnson A, Lewis J, Raff M, Roberts K, Walter P: Molecular Biology of the Cell. 5th edition. New York, U.S.A: Garland Science; 2007.

40. Nelson DL, Cox MM: Lehninger Principles of Biochemistry. New York: W.H. Freeman \& Co; 2005

41. Heiland B, Schneider AS, Arzt E, Weiss IM: Skalenübergreifende Strukturanalyse an Anschliffen und Dünnschliffen von Seeohr-Schalen. In Fortschritte in der Metallographie - Vortragstexte der 13 Internationalen Metallographie-Tagung, 29 September - 01 Oktober 2010 in Leoben. Volume 42. Edited by Kneissl A, Clemens H. Frankfurt, Germany: WerkstoffInformationsgesellschaft mbH; 2010:121-126. Petzow G (Series Editor): Sonderbände der Praktischen Metallographie.

42. Oldenbourg R: Polarized light field microscopy: an analytical method using a microlens array to simultaneously capture both conoscopic and orthoscopic views of birefringent objects. J Microsc 2008, 231:419-432.

43. Nudelman F, Chen HH, Goldberg HA, Weiner S, Addadi L: Spiers memorial lecture. Lessons from biomineralization: Comparing the growth strategies of mollusc shell prismatic and nacreous layers in Atrina rigida. Faraday Discuss 2007, 136:9-25. discussion 107-123.

44. Gilbert PUPA, Young A, Coppersmith SN: Measurement of c-axis angular orientation in calcite $\left(\mathrm{CaCO}_{3}\right)$ nanocrystals using X-ray absorption spectroscopy. Proc Natl Acad Sci USA 2011, 108:11350-11355.

45. Olson IC, Kozdon R, Valley JW, Gilbert PUPA: Mollusk shell nacre ultrastructure correlates with environmental temperature and pressure. J Am Chem Soc 2012, 134:7351-7358.

46. Reimer L: Scanning Electron Microscopy: Physics of Image Formation and Microanalysis. 2nd edition. Berlin, Heidelberg: Springer; 1998.

47. Fratzl P, Gupta HS, Fischer FD, Kolednik O: Hindered crack propagation in materials with periodically varying Young's modulus - Lessons from biological materials. Adv Mater 2007, 19:2657.

48. Perez-Huerta A, Cusack M, Zhu W: Assessment of crystallographic influence on material properties of calcite brachiopods. Miner Mag 2008 72:563-568.

49. Sun J, Bhushan B: Hierarchical structure and mechanical properties of nacre: A review. RSC Adv 2012, 2:7617-7632.

50. Li X, Chang W-C, Chao YJ, Wang R, Chang M: Nanoscale structural and mechanical characterization of a natural nanocomposite material: the shell of red abalone. Nano Lett 2004, 4:613-617.

51. Katti KS, Mohanty B, Katti DR: Nanomechanical properties of nacre. J Mater Res 2006, 21:1237-1242.

52. Fleischli FD, Dietiker M, Borgia C, Spolenak R: The influence of internal length scales on mechanical properties in natural nanocomposites: a comparative study on inner layers of seashells. Acta Biomater 2008, 4:1694-1706.
53. Kearney C, Zhao Z, Bruet BJF, Radovitzky R, Boyce MC, Ortiz C: Nanoscale anisotropic plastic deformation in single crystal aragonite. Phys Rev Lett 2006, 96:255505

54. Mayer G, Sarikaya M: Rigid biological composite materials: Structural examples for biomimetic design. Exp Mech 2002, 42:395-403.

55. Jackson AP, Vincent JFV, Turner RM: The mechanical design of nacre. Proc R Soc Lond B Biol Sci 1988, 234:415.

56. Pokroy B, Demensky V, Zolotoyabko E: Nacre in mollusk shells as a multilayered structure with strain gradient. Adv Funct Mater 2009, 19:1054-1059.

57. Bhimasenachar J: Elastic constants of calcite and sodium nitrate. Proc Math Sci 1945, 22:199-208.

58. Dandekar D: Variation in the elastic constants of calcite with temperature. J Appl Phys 1968, 39:3694.

59. Petzow G: Metallographisches, keramographisches und plastographisches Ätzen. Berlin, Stuttgart: Borntraeger; 2006

60. Schumann H: Metallographie. Stuttgart: Deutscher Verlag für Grundstoffindustrie; 1990

61. Telle R, Petzow G: Light Microscopy. In Materials Science and Technology, Volume 2A. Edited by Cahn RW, Haasen P, Kramer EJ. Weinheim: Verlag Chemie; 1992:357-422

62. Shribak $M$, Oldenbourg $R$ : Techniques for fast and sensitive measurements of two-dimensional birefringence distributions. Appl Opt 2003, 42:3009-3017.

63. Oldenbourg R: Analysis of microtubule dynamics by polarized light. Methods Mol Med 2007, 137:111-123.

64. Oliver WC, Pharr GM: Improved technique for determining hardness and elastic modulus using load and displacement sensing indentation experiments. J Mater Res 1992, 7:1564-1580.

doi:10.1186/2046-1682-5-19

Cite this article as: Schneider et al:: Hierarchical super-structure identified by polarized light microscopy, electron microscopy and nanoindentation: Implications for the limits of biological control over the growth mode of abalone sea shells. BMC Biophysics 2012 5:19.

\section{Submit your next manuscript to BioMed Central and take full advantage of:}

- Convenient online submission

- Thorough peer review

- No space constraints or color figure charges

- Immediate publication on acceptance

- Inclusion in PubMed, CAS, Scopus and Google Scholar

- Research which is freely available for redistribution 